\title{
LA TRANSFERENCIA DE TECNOLOGÍA EN LA EUROPA LATINA: EL PAPEL DE LA SOCIÉTÉ TECHNIQUE DE L'INDUSTRIE DU GAZ EN FRANCE, 1895-1938
}

\author{
Alberte Martínez-López \\ Universidade da Coruña \\ Email: alberte.martinez@udc.es \\ ORCID iD: https://orcid.org/0000-0001-7267-386X \\ Jesús Mirás Araujo \\ Universidade da Coruña \\ Email: jmiras@udc.es \\ ORCID iD: https://orcid.org/0000-0003-3049-1106
}

Recibido: 15 abril 2021; Aceptado: 15 mayo 2021.

Cómo citar este artículo/Citation: Martínez-López, Alberte; Mirás Araujo, Jesús (2021) "La transferencia de tecnología en la Europa Latina: el papel de la Société Technique de l'Industrie du Gaz en France, 1895-1938", Asclepio, 73 (2): p563. https://doi.org/10.3989/ asclepio.2021/21

RESUMEN: Francia, junto con Gran Bretaña, fue el país líder en el nacimiento y consolidación de la industria del gas. Desde mediados del siglo XIX este país desempeñó un papel clave en la difusión de la tecnología de producción de gas de hulla, especialmente en el área mediterránea, en donde concentró un gran volumen de inversión en el exterior. Entre los factores responsables de este proceso debe destacarse la presencia de ingenieros y diversas asociaciones profesionales. Entre estas últimas sobresale la Société Technique de I'Industrie du Gaz en France, una de las principales asociaciones gasistas a nivel internacional. Este organismo, constituido en el año 1874, aglutinaba a profesionales del sector, que actuaron como agentes que pilotaron la transferencia tecnológica internacional. El objetivo del trabajo es analizar la relevancia de la asociación en dicho proceso de difusión técnica, tanto en Francia como en el resto de la Europa latina (España, Italia y Portugal) desde finales del siglo XIX hasta la Segunda Guerra Mundial, empleando las actas de sus congresos como fuente principal.

PALABRAS CLAVE: Gas; Transferencias de Tecnología; Difusión Tecnológica; Sociedades Científicas; Europa Latina.

\section{TECHNOLOGY TRANSFER IN LATIN EUROPE: THE ROLE OF THE SOCIÉTÉ TECHNIQUE DE L'INDUSTRIE DU GAZ EN FRANCE, 1895-1938}

ABSTRACT: France, along with Great Britain, was the leading country in the beginnings and consolidation of the gas industry. Since the mid-nineteenth century, this country played a key role in the diffusion of coal gas production technology, especially in the Mediterranean area, where it concentrated a large volume of foreign investment. Among the underlying factors in this process, the presence of engineers and several professional associations should be highlighted. Among the latter, the Société Technique de I'Industrie du Gaz en France stands out, which was one of the main gas associations at the international level. This organization, established in 1874, brought together professionals from the sector, who acted as agents who drove international technology transfer. The aim of the article is to analyze the relevance of the association in the aforementioned process of technical diffusion, both in France and in the rest of Latin Europe (Spain, Italy, and Portugal) from the late nineteenth century to World War II, using the proceedings of its congresses as the main source.

KEY WORDS: Gas; Technology Transfer; Technological Diffusion; Scientific Societies; Latin Europe. 


\section{INTRODUCCIÓN}

Las transferencias de tecnología desempeñan un papel clave en el crecimiento socioeconómico. Su importancia como factor explicativo del desarrollo se ha consolidado desde el siglo XIX. Dichas transferencias pueden adoptar diversas formas. Tradicionalmente, se ha singularizado la relevancia de los movimientos internacionales de capital, así como de mercancías o procesos. Sin embargo, la circulación de conocimiento desempeñó un papel de primer orden, de manera directa (publicaciones, exposiciones, desplazamiento de científicos, etc.) o a través de otros canales menos visibles. Es en esos otros medios en donde se centra el presente trabajo. En las vías indirectas de transferencia de conocimiento, a través de las vinculaciones que se desarrollaron entre miembros de la comunidad científica de distintos países. Más concretamente, a través de diversas asociaciones profesionales que permitían el intercambio de ideas, información, etc. entre sus miembros. Esa interconexión se vio favorecida por la internacionalización de las grandes compañías que intervinieron en la construcción y modernización de las infraestructuras de servicios públicos. El centro de atención se desplaza hacia un sector concreto, el de las redes de energía, en particular el gas, cuya expansión se aceleró durante la segunda industrialización. En dicho proceso tuvo especial trascendencia Francia, una de las naciones pioneras en la internacionalización del capital y el conocimiento, así como en el desarrollo del sector de producción y distribución de gas de hulla, en especial en el área mediterránea, en donde concentró una elevada fracción de su inversión exterior.

En este país se constituyó en 1874 la Société Technique de l'Industrie du Gaz en France, una asociación que, bajo una denominación diferente, sigue todavía funcionando en la actualidad. Este organismo aglutinaba a profesionales del sector, que actuaron como agentes que pilotaron la transferencia tecnológica internacional. El objetivo es analizar la importancia de la asociación en la difusión de la tecnología gasista en la Europa latina (Francia, España, Italia y Portugal) en el período delimitado por el inicio de la competencia eléctrica y de la Segunda Guerra Mundial, empleando como fuente principal las memorias de sus congresos.

\section{DIFUSIÓN TECNOLÓGICA, INVERSIÓN EXTERIOR Y CRECIMIENTO ECONÓMICO}

La tecnología constituye un factor clave en el progreso material de una sociedad. La mayoría de economistas e historiadores económicos contempla el cambio tecnológico como uno de los vectores principales en los procesos de crecimiento (Helpman, 2004). No se trata de un fenómeno reciente, ya que tras su irrupción durante la industrialización, tecnología, ciencia e innovación - y uno de sus derivados, la circulación de conocimiento - se convirtieron en elementos clave de los nuevos paradigmas económicos.

Los modelos explicativos se han centrado en dos clases de cambio técnico: la innovación obtenida a partir de la investigación y el desarrollo, y la transferencia y difusión de la tecnología a través de la asimilación y adaptación de tecnología extranjera avanzada. La mayoría de los estudios empíricos iniciales se centraron en el primer elemento. El fuerte impulso de las inversiones internacionales en las últimas décadas ha atraído un creciente interés de los investigadores por los mecanismos de difusión de la tecnología (Woo, 2009).

La inversión extranjera constituye uno de los vehículos principales de difusión de nuevas tecnologías, con especial relevancia en las primeras fases de la industrialización, en las que los contrastes entre los focos emisores y los receptores (centro y periferia) resultan más notorios (De Mello, 1997). No obstante, en los últimos años se ha cuestionado la incidencia de la inversión exterior directa en dicho proceso o su impacto en las economías receptoras, debido a las limitaciones de éstas en su capacidad de absorción, principalmente por la escasa formación de sus trabajadores (Van Pottelsberghe y Lichtenberg, 2001), aunque el debate no está cerrado.

El término transferencia de tecnología nace como una derivación de los estudios sobre el desarrollo (Edgerton, 1999, p. 2). Aunque los expertos definen el concepto de maneras muy diferentes, existe consenso en considerar que es el movimiento de una tecnología, producto o conocimiento, desde un individuo u organización hacia otro distinto (Inkster, 1991, p. 20). Existe un debate en torno al potencial de innovación que se puede generar cuando la transmisión se realiza entre países. Es necesario tener en cuenta que la innovación autóctona (nacional) no ha sido la fuente principal de tecnología de un país, sino que la transferencia de tecnología entre países ha generado impactos muy positivos. Aunque para numerosos autores la transmisión entre países ricos es la más común y relevante, lo cierto es que cuando ésta se efectúa desde un país avanzado hacia otro periférico, causa procesos de innovación importantes, cuyo potencial se eleva cuanto mayor es la distancia tecnológica entre el país líder y el seguidor (Cubel et al., 2012, p. 114). De ahí que la transición hacia el capitalismo industrial en las naciones más atrasadas fuese más dependiente de las tecnologías extranjeras. 
Los especialistas en historia de la tecnología tienden a rehuir del determinismo tecnológico (Berg y Bruland, 1998, pp. 3, 10-11). El paradigma tradicionalmente dominante ponía el acento en los generadores de los cambios (ingenieros, inventores, técnicos) (Pretel, 2009 , p. 60). Sin embargo, desde finales del siglo pasado se contempla el cambio tecnológico de una manera más amplia, como parte de un proceso interrelacionado y complementario de invenciones o adaptaciones, en el que tienen cabida otros diversos factores explicativos, y no exclusivamente los puramente tecnológicos. Las nuevas aproximaciones conceptuales tienen una marcada influencia de las investigaciones sobre sociología de la ciencia y la tecnología. Consideran que el cambio tecnológico es acumulativo y poliédrico (Cantwell, 1990), y guarda una estrecha relación con variables contextuales, que incluyen factores económicos, institucionales, sociales, culturales o políticos (Berg y Bruland, 1998, p. 3).

Al contemplar la transferencia de tecnología desde una perspectiva más amplia, se tiene en cuenta no sólo la generación de innovaciones sino la capacidad de adopción de las nuevas tecnologías. El desarrollo tecnológico es el resultado de una elaboración colectiva, por lo que es necesario un ambiente social receptivo (Mokyr, 1992, p. 325). Abramovitz acuñó el término capacidad social de absorción, que tendría que ver directamente con la capacitación técnica y la cualificación del trabajo existente en una sociedad, y que incluye como elemento central la extensión del período de educación, junto con la acción de diversas instituciones políticas, comerciales, industriales o financieras. La absorción de la tecnología procedente de los países líderes choca, asimismo, con lo que denomina "congruencia tecnológica" (Abramovitz, 1979), que incluye condiciones económicas, tales como el tamaño del mercado o la escala de la producción en la que la nueva tecnología se va a asentar.

Los canales a través de los que se realiza la transferencia y, por tanto, la innovación a través de nuevas tecnologías, son diversos. Los más importantes son las inversiones directas de capital, las patentes y los acuerdos para la cesión de licencias, la creación de empresas, los intercambios comerciales (básicamente, la importación de bienes y tecnología), los desplazamientos de capital humano (principalmente empresarios y profesionales) o el espionaje industrial. Desde el siglo $\mathrm{XIX}$, estas formas se han mantenido relativamente estables, aunque algunas de ellas hayan experimentado importantes desarrollos durante las últimas décadas (multinacionales).
Sin embargo, nos interesa destacar en este texto un tipo concreto de agentes de transferencia, los científicos e ingenieros, cuyo papel fue decisivo, a través de una doble vía. Por un lado, su intervención como agentes directos de transmisión del conocimiento. Por otro, gracias a los vínculos que mantuvieron con la comunidad científica de otros países, mediante las publicaciones que realizaban o difundían, los viajes de estudio o a exposiciones internacionales al extranjero, su pertenencia a redes intelectuales, a comunidades científicas y a asociaciones profesionales, o su emigración a otros países (Ortiz-Villajos, 2006, pp. 54-55). Esa participación se vio favorecida por la internacionalización de las grandes compañías que intervinieron en la construcción y modernización de las empresas de servicios públicos en otros países.

Desde el siglo XIX, el mercado energético se ha caracterizado por su naturaleza transnacional, ya que sus dinámicas han sobrepasado las fronteras estatales. En ese sentido, la inversión extranjera directa realizada por las multinacionales desempeñó un papel crucial en la difusión de la tecnología, lo que incluye la transmisión de conocimiento a través de distintos canales. Concretamente, como señala Martykánová (2010, pp. 24-25), una corriente dentro de la historiografía sobre la ciencia y la tecnología ha subrayado la trascendencia de los ingenieros en la circulación del conocimiento, a través de su inserción en las redes intelectuales.

Durante los primeros años del siglo XIX, el país pionero en la difusión de conocimiento fue Gran Bretaña, sobre todo gracias a las transferencias realizadas por los ingenieros de este país (Buchanan, 1986). Francia se incorporó algo más tarde a esta corriente, desde aproximadamente las décadas de 1840 y 1850 (Williot, 2006). Sin embargo, a partir de la llegada de la segunda industrialización el proceso se agudizó, alcanzando una escala global (Inkster, 1998, p. 309). Desde los años ochenta y durante el primer tercio del siglo XX, ingenieros, científicos $y$, en menor medida, técnicos especializados se convirtieron en la principal fuente de innovación y transferencia de tecnología, creándose y expandiéndose redes de innovación, a través de los vínculos establecidos entre ingenieros y técnicos de los países desarrollados con empresarios de los países de la periferia (Sáiz, 2012, p. 365). Además, se incrementó la nómina de naciones involucradas en la circulación de capital y de conocimiento, extendiendo el abanico hacia otros países, como Alemania y Estados Unidos.

Interesa destacar el caso de Francia, en la medida en que la asociación objeto de análisis tuvo su origen en ese país. En el caso concreto de la industria del 
gas, desde mediados del siglo XIX, Francia se halló en disposición de exportar el conocimiento adquirido, a través de un doble movimiento, financiero (capital) y técnico. En el plano técnico, los ingenieros franceses se convirtieron en elementos imprescindibles en la introducción de la tecnología de manufacturación del gas en Europa, principalmente en los países de la cuenca mediterránea (Williot, 2006, pp. 207-209). Hasta los años sesenta, fueron químicos o farmacéuticos los responsables de la innovación, pero posteriormente pasaron a ser ingenieros civiles.

Desde el último cuarto del siglo XIX, la movilidad internacional se incrementó, por efecto de la difusión de capital y la revitalización de una profesión de ingeniero en pleno proceso de transformación como consecuencia de la reforma de las enseñanzas de ingeniería en Francia (Grelon, 1993, pp. 42-43). En cambio, durante el período de entreguerras la inversión extranjera se redujo substancialmente, afectada por los trastornos financieros y el creciente clima nacionalista. En España, por ejemplo, la inversión gasista extranjera casi desapareció por impacto de la guerra europea. En paralelo, se produjo una importante concentración empresarial, con la formación de potentes grupos energéticos liderados por la electricidad.

\section{LA INDUSTRIA DEL GAS EN LA EUROPA LATINA}

La irrupción de la tecnología del gas tuvo una gran trascendencia en las sociedades avanzadas del siglo XIX. Conllevó una auténtica revolución social, pues contribuyó a una profunda transformación del modo de vida de sus habitantes, pero también tuvo un notable impacto económico. El crecimiento industrial y la expansión urbana hicieron que se convirtiese en una de las industrias en red más importantes (Tomory, 2012, p. 5), siendo, además, pionera en anticipar la relación entre la ciencia y la tecnología, lo que andando el siglo constituirá la base de la segunda industrialización.

La industria del gas tuvo su origen en Gran Bretaña y Francia hacia la última década del siglo XVIII. Su desarrollo comercial comenzó en Londres, la primera ciudad que disfrutó de un suministro regular desde 1812. Durante el despegue del sector, la tecnología y el conocimiento eran británicos, siendo éstos quienes promovieron su expansión hacia Europa (Tomory, 2011, p. 396), si bien en el continente se difundieron también métodos de destilación franceses.

Su uso se concentró inicialmente en la iluminación, al menos hasta las décadas de 1850-1860. Las redes de distribución eran locales, desempeñando un papel clave los umbrales de demanda, ya que la rentabilidad del servicio se hallaba directamente relacionada con el tamaño del mercado. Aunque la expansión continental fue más lenta, las ciudades más importantes de cada país comenzaron pronto a adoptar el gas como sistema de alumbrado de sus calles, generalizándose en pocas décadas. En su difusión se pueden establecer varias fases.

En la primera, hasta 1840 , su alcance se redujo a las naciones líderes (Gran Bretaña y Francia) y Bélgica (Bruselas inauguró su servicio en 1819), esta última sintomáticamente próxima a aquellas y bien dotada de carbón.

Entre 1840 y 1910 se produce la difusión del gas en sucesivos círculos geográficos y económicos: Europa central (sobre todo, Alemania y Suiza, donde alcanzó una penetración más dilatada) y nórdica (Thomas, 2018, pp. 146-156). En estos países, las ciudades grandes contaban con suministro de gas desde los años veinte y treinta (Berlín, 1826), pero lo sustancial es que las ciudades pequeñas y medianas dispusieron del servicio de manera generalizada desde los sesenta.

Con algo más de retraso se sitúan aquellos países en los que el gas sólo llegó a las grandes ciudades hacia la década de 1860, aunque algunas urbes de mayor tamaño comenzaron a implementarlo hacia los años cuarenta. Es el caso de Italia, España y Portugal. En general, el período que transcurre entre las décadas de 1840 y 1880 constituye una etapa de esplendor para la industria gasista en estos países. Las causas fueron el crecimiento y modernización de las ciudades, las mejoras en los procesos de fabricación y distribución y la ausencia de competencia de otras energías en la iluminación.

Dentro del bloque de la Europa latina, Francia fue la nación más avanzada en la introducción y difusión del gas. La cronología de su implantación muestra una primera oleada en las décadas de 1820 y 1830, cuando fueron iluminadas las ciudades más importantes del país (Williot y Paquier, 2005a, p. 24). A continuación, experimentó un período de prosperidad entre el comienzo del Segundo Imperio (1852) y la llegada de la electricidad en los años ochenta, cuyo impulso se halla en la demanda generada por la urbanización y el inicio en los años cuarenta de los proyectos de renovación urbanística, que tendrían un nuevo impulso desde los setenta. París fue testigo de las primeras iniciativas (entre 1817 y 1822 se fundaron cuatro empresas), actuando como catalizador de la propagación del gas. En 1840, 35 ciudades habían otorgado una concesión, y 
en 1850 el número de municipios atendidos aumentó a 107. En 1891, todos los municipios de más de 20.000 habitantes se hallaban conectados y casi la mitad de los de menos de 2.000 habitantes disponían de conexión (Williot, 2005, pp. 147-160).

Con un desfase de unas dos décadas el gas desembarcó en las penínsulas ibérica e italiana. A pesar de la dificultad para dibujar un modelo homogéneo para todo su territorio, Italia fue la segunda nación más adelantada de este grupo, con algunas experiencias relativamente tempranas, concentradas en el norte y centro del país. La primera autorización se produjo en Turín (1837). En la década de 1840 las primeras ciudades comenzaron a reemplazar el arcaico alumbrado por aceite, difundiéndose en los cincuenta y sesenta. Llama la atención la tardía penetración en Roma (1854), la última entre las capitales europeas. Al inicio de la Unificación todas las ciudades grandes y medianas disponían de redes gasistas, aunque la penetración en las pequeñas urbes apenas había comenzado. En 1865 había 57 fábricas de gas y para 187055 ciudades disponían de alguna instalación (Franco, 1988, pp. 29-30). Los siguientes veinte años que transcurrieron hasta la aparición de la electricidad fueron el momento del despegue definitivo de un sector decisivo en la modernización urbana (Giuntini, 1997, pp. 166-167).

En España, a pesar de que existe constancia de algunos ensayos de alumbrado de gas relativamente tempranos, la difusión fue más tardía, lenta y modesta. En algunas ciudades, su entrada se demoró hasta los años ochenta y en bastantes más se produjo directamente la transición desde los sistemas tradicionales de alumbrado hacia la electricidad, sin el paso intermedio del gas. Su despegue data de 1842, fecha del primer contrato en Barcelona. Esta fase inicial se prolonga hasta aproximadamente 1861, fecha en la que operaban 28 fábricas. El período posterior, 1861-1901, será el de construcción del mayor número de instalaciones, incrementándose a 81 el total de fábricas en funcionamiento (Alayo y Barca, 2017, pp. 144, 318-320). Una característica específica española es la diferenciación de dos modelos gasistas, el del conjunto del Estado y el de Cataluña, más cercano a los estándares europeos avanzados.

Finalmente, Portugal se aproxima al patrón español, aunque con una menor profundidad territorial. El gas comenzó a extenderse desde finales de la década de 1840, pero sólo en las ciudades más grandes: Lisboa (1848), Oporto (1855) y Coimbra (1856). El rasgo más significativo es el largo lapso de tiempo transcurrido (al menos cuatro décadas) entre la instalación en estos núcleos y los demás centros urbanos. En los ochenta, década de mayor difusión, cinco nuevas ciudades adoptaron este sistema de alumbrado. En 1896 apenas había 11 fábricas, las mismas que en 1907 (Cardoso, 2017, pp. 77-78). El principal problema de la tardía adopción del gas fue la escasez de demanda, ligada a los reducidos niveles de renta, lo que explica el fracaso de algunas iniciativas o que se perdieran algunas concesiones, por imposibilidad de ponerlas en funcionamiento.

Dentro del proceso de expansión descrito varios elementos resultan cruciales. Por el lado de la oferta, la disponibilidad de capitales, conocimientos y materia prima (hulla) a precios competitivos, así como las posibilidades de obtención y venta de subproductos. Estos países adolecían de una pobre dotación de carbón, por lo que dependían del de importación, lo que explica que algunas de las primeras redes se trazasen en ciudades portuarias o con acceso a vías de transporte alternativas (ferrocarril).

A eso se sumaron las carencias de capital, capital humano, experiencia empresarial y tecnología. Todo ello posibilitó la rápida penetración de empresas privadas extranjeras (multinacionales), que desempeñaron un papel crucial en el desarrollo inicial del sector. En España y Portugal (en donde la presencia foránea fue más relevante) predominó el capital francés, belga y británico. La principal excepción la constituyó Cataluña, en donde el peso de los promotores locales fue muy elevado. En Italia se añadía a esa nómina también el capital suizo y el alemán.

Fue necesario, asimismo, establecer el marco regulador bajo el que deberían operar las compañías. El servicio fue acometido por empresas privadas, que operaron bajo la figura jurídica de la concesión, normalmente en situación de monopolio. Al contrario que en Gran Bretaña o Alemania, en donde tuvo mayor fuerza la municipalización, fueron poco frecuentes los casos de asunción directa del servicio por parte de los ayuntamientos, con la excepción de Italia en los años previos a la guerra europea.

Desde la demanda eran necesarios varios elementos. Por un lado, un cierto nivel de desarrollo industrial, que permitiese unos niveles de renta mínimos que justificasen las elevadas inversiones que requería una costosa infraestructura en red. Por otro, unas tasas de urbanización mínimas, dado que se trata de un servicio localizado en las ciudades. El menor nivel de desarrollo alcanzado explica la cronología y el menor grado de penetración del gas y el destacado papel de los países pioneros en su difusión. 
En las dos últimas décadas del siglo XIX surgió una seria competencia para la industria del gas, la electricidad, aunque la transición no fue inmediata ni abrupta, sino que el gas todavía experimentó importantes crecimientos durante las dos siguientes décadas. La presencia de la nueva energía actuó de catalizador para la creación de grupos empresariales que combinaban el gas y la electricidad, dentro de una nueva oleada expansiva de los movimientos internacionales de capital. Estos cambios coincidieron con una modernización tecnológica de la industria gasista y con una diversificación de sus usos (cocina, calentadores de agua, motores), que permitió su sostenimiento entre los últimos años del siglo XIX y principios del XX (Williot y Paquier, 2005b, pp. 56-60). En cambio, el gas perdía presencia en el mercado de alumbrado, mientras la electricidad avanzaba a una velocidad ascendente. La producción de origen hidráulico, unida a la transmisión a distancia de la energía, fortalecieron definitivamente la posición de la electricidad en los mercados más importantes, los urbanos (Hausman, Hertner y Wilkins, 2008, p. 12).

Sin embargo, antes de la Primera Guerra Mundial, la electricidad todavía no había conseguido desplazar al gas. El conflicto fue el momento más crítico para esta industria, del cual ya no se recuperaría. A pesar de que durante el período de entreguerras el gas todavía continuó expandiéndose, la electricidad acrecentó su dominio durante los años veinte. A la altura de la Segunda Guerra Mundial la industria no había conseguido modernizarse y racionalizarse de manera eficiente. El sector adolecía de problemas estructurales (fragmentación, reducida escala, falta de coordinación ...) que erosionaban su posición competitiva. Estaba a punto de inaugurarse una nueva era, con la transición hacia el gas natural o el gas de nafta (además del petróleo), que tendría lugar con posterioridad al conflicto.

\section{LA SOCIÉTÉ TECHNIQUE DE L'INDUSTRIE DU GAZ EN FRANCE EN EL PRIMER TERCIO DEL SIGLO XX}

La Société technique de l'industrie du gaz en Fran$c e^{1}$ se fundó en 1874 , como resultado de la confluencia de los dos polos gasistas franceses más potentes, el de París y el de Lyon. Su nacimiento se enmarca en la eclosión de sociedades profesionales gasistas que tuvo lugar, a escala internacional, durante la década de los sesenta y, sobre todo, setenta del siglo XIX (Braunholtz, 1963). Su influencia superó ampliamente el marco francés, constituyendo la asociación sectorial europea más potente (Fàbregas, 2003, p. 83).

Hasta 1892, la sociedad fue el único organismo representativo del sector ante los poderes públicos y la sociedad. Ese año, la patronal gasista creó un organismo específico para la defensa de sus intereses, el Syndicat professionnel de l'industrie du gaz. Hasta 1927 ambas entidades coexistirán, sin apenas contactos e incluso, en ocasiones, con ciertas tensiones.

Luego de un estancamiento de la sociedad durante los años 90 debido a la aparición y desarrollo de la nueva tecnología eléctrica, a lo largo del primer tercio del siglo XX se produjo un relanzamiento de la sociedad, manifestado en un fuerte crecimiento del número de socios, que se duplica, seguramente en relación con la redefinición de la industria del gas. Este impulso se ve acompañado por una mayor imbricación, en número y financiación, con el tejido empresarial, con una parte significativa de él ubicado en el exterior (Tabla 1).

Tabla 1. Socios, congresistas y subvenciones de la Société technique de l’industrie du gaz en France, 1895-1938

\begin{tabular}{|c|c|c|c|c|c|}
\hline Año & Socios & Congresistas & $\begin{array}{c}\text { Subvenciones, en francos, } \\
\text { de las compañías de gas }{ }^{(1)}\end{array}$ & $\begin{array}{c}\text { Compañías } \\
\text { patrocinadoras }\end{array}$ & $\begin{array}{c}\text { Compañías patrocinadoras } \\
\text { extranjeras o en el extranjero }\end{array}$ \\
\hline 1895 & 611 & 240 & 21.380 & 32 & 66 \\
\hline 1914 & 879 & 369 & 33.814 & 11 \\
\hline 1929 & 996 & & 14.943 & & 6 \\
\hline
\end{tabular}

Fuente: Société technique de l'industrie du gaz en France, Compte rendu du congrès... ${ }^{(1)}$ A partir de 1929 la subvención procede de I’Union Syndicale. Datos en francos oro de 1913, elaboración propia. 
La participación más o menos activa de los socios en forma de asistencia a los congresos anuales de la asociación crece a lo largo del tiempo en términos absolutos y también ligeramente en términos relativos, pasando del $39 \%$ al $42 \%$ entre 1895-1914. de influencia francesa. Sorprende la escasa presencia de alemanes, otro gran país gasista, aunque quizás la existencia de una potente asociación propia en ese país y la tensión entre ambos países en esos años de la Paz armada lo expliquen (Tabla 2).

Tabla 2. Miembros de la Société technique de l'industrie du gaz en France, por países y porcentajes, año 1895

\begin{tabular}{|c|c|c|c|}
\hline Países & Miembros & Países & Miembros \\
\hline Francia & $487(79,7 \%)$ & Austria & $2(0,3 \%)$ \\
\hline España & $27(4,4 \%)$ & Brasil & $2(0,3 \%)$ \\
\hline Bélgica & $25(4,1 \%)$ & Estados Unidos & $2(0,3 \%)$ \\
\hline Gran Bretaña & $25(4,1 \%)$ & Grecia & $2(0,3 \%)$ \\
\hline Italia & $10(1,6 \%)$ & Madagascar & $2(0,3 \%)$ \\
\hline Suiza & $4(0,7 \%)$ & Portugal & $2(0,3 \%)$ \\
\hline Holanda & $4(0,7 \%)$ & Rumanía & $1(0,2 \%)$ \\
\hline Argelia & $4(0,7 \%)$ & Argentina & $1(0,2 \%)$ \\
\hline Egipto & $4(0,7 \%)$ & Irlanda & $611(100 \%)$ \\
\hline Rusia & $3(0,5 \%)$ & & TOTAL \\
\hline Alemania & $2(0,3 \%)$ & & \\
\hline
\end{tabular}

Fuente: Société technique de l'industrie du gaz en France, Compte-rendu du vingt deuxième congrès, Paris, 1895, pp. 605-622. Elaboración propia.

Como cabría esperar de una asociación francesa, la mayoría de sus miembros procedía de este país. No obstante, una quinta parte de los mismos eran extranjeros, lo que evidencia su vocación internacional. La procedencia era bastante diversificada, desde Europa a América o África, aunque predominaban los países cercanos como Gran Bretaña, Bélgica (histórica y lingüísticamente conectada con Francia) y países de la Europa latina como España e Italia. También era significativa la presencia de socios del norte de África, zona
Aparte de la presencia como socios y en los congresos de profesionales procedentes de otros países, es importante recalcar el papel de los ingenieros franceses que gestionaban fábricas en el extranjero en la transmisión de los conocimientos tecnológicos del sector.

En 1914 la composición interna de la entidad mantenía los rasgos generales anteriores, pero con ciertos matices significativos (Tabla 3). Se estaba perdiendo

Tabla 3. Miembros de la Société technique de l'industrie du gaz en France, por países y porcentajes, año 1914

\begin{tabular}{|c|c|c|c|}
\hline Países & Miembros & Países & Miembros \\
\hline Francia & $740(84,5 \%)$ & Grecia & $3(0,3 \%)$ \\
\hline Bélgica & $40(4,6 \%)$ & Rusia & $2(0,2 \%)$ \\
\hline Reino Unido & $32(3,7 \%)$ & Argelia & $1(0,1 \%)$ \\
\hline España & $19(2,2 \%)$ & Alemania & $1(0,1 \%)$ \\
\hline Italia & $15(1,7 \%)$ & Portugal & $1(0,1 \%)$ \\
\hline Suiza & $9(1,0 \%)$ & Rumanía & $1(0,1 \%)$ \\
\hline Egipto & $3(0,3 \%)$ & Turquía & $876(100 \%)$ \\
\hline Estados Unidos & $3(0,3 \%)$ & & TOTAL \\
\hline Holanda & $3(0,3 \%)$ & & \\
\hline
\end{tabular}

Fuente: Société technique de l'industrie du gaz en France, Compte-rendu du quarante et unième congrès, Paris, 1914, pp. 788809. Elaboración propia. 
algo la impronta internacional, manifestado en el aumento del predominio francés y en la reducción del número de países representados, en especial de los americanos. Ello podría estar en relación con el enrarecimiento de las relaciones internacionales, que también afectaba a los intercambios profesionales, en vísperas de la guerra. También se aprecia una importante reducción en la presencia española, que pasa del 20 lugar al 4으, compensada por el aumento de Italia y Suiza. El retroceso español podría estar explicado por la progresiva sustitución de técnicos franceses por nativos en las fábricas de gas de España y la creciente incidencia de la vía nacionalista del capitalismo español, que se manifestaría en un cierto repliegue interior de los profesionales españoles.

La asociación se regía por un comité de doce miembros, un Consejo y un Secretariado. Como apoyo a los trabajos del comité se crearon comisiones, inicialmente puntuales para el estudio de determinados temas concretos, y que a partir de 1910 se convirtieron en permanentes.

Durante las primeras décadas fueron desempeñando la presidencia los principales prohombres/dinastías de la industria gasista francesa, como Mallet, Vautier, Ellisen, Lebon o Lachomette, que por extensión lo eran también de la europea, lo que evidencia la importancia de la asociación. No obstante, esa presencia se diluye a partir de principios del siglo $X X$, probablemente en relación con la creación en 1892 de la patronal gasista y la consecuente separación de funciones entre ambas entidades, lo que llevará a una mayor profesionalización de la Sociétè (Tabla 4)².
En 1927 la Société Technique inaugura una nueva etapa, refundándose, bajo la nueva fórmula jurídica de sindicato profesional, como Association Technique du Gas, constituyendo con la entidad patronal citada supra l'Union syndicale de l'industrie du gaz. Intensificará sus actividades (Congresos anuales, comisiones, premios y concursos, formación profesional, servicio de documentación ${ }^{3}$, estandarización), dotándolas de mayor eficacia ${ }^{4}$. En la esfera internacional, la ATG, de la mano de Auguste Baril (presidente) y Pierre Mougin (secretario), desempeñará un papel clave en la creación en 1931 de la Union internationale de l'industrie du gaz. De hecho, Baril ostentará su presidencia en el trienio 1934-1937, celebrándose en este último año el congreso trienal de la Union en París ${ }^{5}$.

En vísperas de la crisis de 1929 la sociedad continúa su marcha ascendente, manifestada en el incremento del número de socios, que ronda el millar, y diversifica la procedencia de los mismos, que alcanza ahora los 21 países, aunque el país anfitrión, Francia, aumenta ligeramente su peso (Tabla 5). EEUU incrementa substancialmente su presencia, apareciendo también Japón, frente a un pequeño retroceso de los viejos países industriales europeos como Bélgica, Gran Bretaña o Italia, indicativo de los cambios económicos en la posición relativa de los países derivados del impacto de la Gran Guerra.

Curiosamente, la depresión de los años treinta no parece haber hecho mella en la asociación, pues continúa el aumento de socios, un $20 \%$, y de países representados, 24 (Tabla 6). Los países cercanos a Francia siguen manteniendo un peso significativo, con un fuerte ascenso de España, que pasa del 40 al $2 \circ$ puesto, frente a una Gran Bretaña que acentúa su decadencia.

Tabla 4. Presidentes de la Société technique de l'industrie du gaz en France, 1895-1939

\begin{tabular}{|c|c|c|c|}
\hline Prosper de Lachomette & $1894-1896$ & Henri Laurain & $1919-1920$ \\
\hline Philibert Delahaye & $1896-1898$ & Edouard Kaeuffer & $1920-1921$ \\
\hline Théodore Vautier & $1898-1900$ & Robert Ellissen & $1921-1923$ \\
\hline Alfred Lebon & $1900-1901$ & Francis Rouland & $1923-1924$ \\
\hline Maurice Piaton & $1902-1903$ & Lucien Rolland & $1924-1927$ \\
\hline Jules Deleury & $1903-1904$ & Auguste Baril & $1927-1929$ \\
\hline Georges Visinet & $1905-1906$ & Hippolyte Laedlein & $1929-1930$ \\
\hline André Coze & $1906-1908$ & Auguste Sellié & $1930-1933$ \\
\hline Auguste Godinet & $1908-1910$ & Léon Carpentier & $1933-1935$ \\
\hline Henri Marquisan & $1910-1912$ & Alfred Bazille & $1935-1937$ \\
\hline Auguste Boutan & $1912-1914$ & Louis Stoss & $1937-1939$ \\
\hline Francis Rouland & $1914-1919$ & & \\
\hline
\end{tabular}

Fuente: ATIGF (1974, pp. 35-36). 
Tabla 5. Miembros de la Association technique de l'industrie du gaz en France, por países y porcentajes, año 1929

\begin{tabular}{|c|c|c|c|}
\hline Países & Miembros & Países & Miembros \\
\hline Francia & $851(85,4 \%)$ & Turquía & $1(0,2 \%)$ \\
\hline Bélgica & $38(3,8 \%)$ & Alemania & $1(0,1 \%)$ \\
\hline Gran Bretaña & $29(2,9 \%)$ & Brasil & $1(0,1 \%)$ \\
\hline España & $23(2,3 \%)$ & Canadá & $1(0,1 \%)$ \\
\hline Argelia & $10(1,0 \%)$ & Dinamarca & $1(0,1 \%)$ \\
\hline Estados Unidos & $10(1,0 \%)$ & Egipto & $1(0,1 \%)$ \\
\hline Suiza & $7(0,7 \%)$ & Grecia & $1(0,1 \%)$ \\
\hline Italia & $6(0,6 \%)$ & Polonia & $1(0,2 \%)$ \\
\hline Checoslovaquia & $5(0,5 \%)$ & Túnez & $1(0,1 \%)$ \\
\hline Holanda & $4(0,4 \%)$ & Yugoslavia & $996(100 \%)$ \\
\hline Japón & $2(0,2 \%)$ & TOTAL & \\
\hline
\end{tabular}

Fuente: Association technique de l'industrie du gaz en France, Compte-rendu du cinquante-deuxième congrès de l'industrie du gaz, Paris, 1929, pp. 439-451. Elaboración propia.

En el capítulo de la formación cabe destacar la creación por la Société technique del Centre d'études supérieures gazieres en 1930, dirigido a la formación específica de los ingenieros de esta industria, con gran éxito y presencia habitual de estudiantes extranjeros ${ }^{6}$. Con anterioridad, en 1921, se había puesto en marcha la Semana gasista, con una programación de carácter generalista. Para facilitar la formación a distancia, en especial de cuadros medios de pequeñas fábricas de provincias, se iniciaron cursos profesionales, de tres años de duración, por correspondencia en 1935, que alcanzaron también una notable repercusión.

Aunque se trataba de una asociación francesa, se mantenían flujos de información internacional a través de los socios y congresistas extranjeros, las asociacio-

Tabla 6. Miembros de la Association technique de l'industrie du gaz en France, por países y porcentajes, año 1938

\begin{tabular}{|c|c|c|c|}
\hline Países & Miembros & Países & Miembros \\
\hline Francia & $1.074(89,4 \%)$ & Canadá & $1(0,1 \%)$ \\
\hline España & $27(2,2 \%)$ & Dinamarca & $1(0,1 \%)$ \\
\hline Bélgica & $23(1,9 \%)$ & Egipto & $1(0,1 \%)$ \\
\hline Gran Bretaña & $19(1,6 \%)$ & Grecia & $1(0,1 \%)$ \\
\hline Argelia & $10(0,8 \%)$ & Japón & $1(0,1 \%)$ \\
\hline Estados Unidos & $9(0,7 \%)$ & Luxemburgo & $1(0,1 \%)$ \\
\hline Suiza & $9(0,7 \%)$ & Polonia & $1(0,1 \%)$ \\
\hline Italia & $8(0,7 \%)$ & Rumanía & $1(0,1 \%)$ \\
\hline Checoslovaquia & $4(0,3 \%)$ & Túnez & $1(0,1 \%)$ \\
\hline Holanda & $3(0,2 \%)$ & Turquía & $1(0,1 \%)$ \\
\hline Alemania & $2(0,2 \%)$ & Yugoslavia & $1.201(100 \%)$ \\
\hline Austria & $1(0,1 \%)$ & & TOTAL \\
\hline Brasil & $1(0,1 \%)$ & & \\
\hline
\end{tabular}

Fuente: Association technique de l'industrie du gaz en France, Compte-rendu du soixante et unième congrès de l'industrie du gaz, Paris, 1938, pp. 509-522. Elaboración propia. 
nes profesionales de otros países y las revistas científicas internacionales. El Journal des usines a gaz, iniciado en 1877 y de periodicidad mensual, era el órgano oficial de la asociación. Sus abonados oscilaban en torno a los 500-550 hasta la Primera Guerra Mundial, un centenar de los cuales residían en el extranjero, pasando en 1935 a 900 abonados, 250 de ellos extranjeros, lo que evidencia su impronta en la difusión internacional del conocimiento gasista.

A lo largo de esta etapa, y en función de los puntos de interés de cada momento, la asociación hizo énfasis en diversos temas (Tabla 7). Como constante, la temática predominante fueron los procesos de fabricación del gas (destilación, depuración, hornos, gasómetros), con carácter abrumador a partir del carbón, aunque con cierta presencia de otras materias primas (gas de agua, etc.), seguramente estimulada por la creciente competencia de la electricidad. El análisis de los subproductos (coque, alquitrán, aguas amoniacales, etc.) mantuvo un constante, aunque lógicamente menor, interés. da también, en este caso, por la aparición de la electricidad y la consiguiente necesidad de búsqueda de nuevos nichos de mercado para la industria gasista. En este último aspecto, resulta significativo el importante número de comunicaciones dedicadas específicamente a estudiar la electricidad.

Los aspectos laborales también tuvieron cierta acogida en los congresos, centrándose las discusiones principalmente en la mejora de la productividad de los operarios, a través de la formación profesional y el establecimiento de incentivos, el retiro obrero y la seguridad laboral. Finalmente, en el capítulo "otros" figurarían principalmente estudios globales sobre la industria del gas y análisis de la legislación relacionada con esta actividad. No obstante, es interesante destacar cómo desde los años noventa la temática congresual se centra casi exclusivamente en aspectos técnicos, probablemente por la creación del Syndicat professionnel en 1892, al que se le reservarían los temas laborales, jurídicos y genéricos.

Tabla 7. Temática de las comunicaciones de los congresos de la Société technique, 1894-1938, en porcentaje

\begin{tabular}{|c|c|c|c|c|c|c|}
\hline & $\mathbf{1 8 9 4 - 1 8 9 8}$ & $\mathbf{1 8 9 9 - 1 9 0 3}$ & $\mathbf{1 9 0 4 - 1 9 0 6}$ & $\mathbf{1 9 1 4}$ & $\mathbf{1 9 2 9}$ & $\mathbf{1 9 3 8}$ \\
\hline Producción & 30,8 & 39,4 & 40,5 & $\mathbf{2 8 , 6}$ & 56,3 & 33,3 \\
\hline Subproductos & 6,6 & 5,3 & 2,4 & 9,5 & 6,3 & 8,3 \\
\hline Distribución & 23,1 & 13,6 & 13,1 & $\mathbf{2 8 , 6}$ & 6,3 & 8,3 \\
\hline Alumbrado & 22,0 & 25,0 & 27,4 & 9,5 & 0,0 & 8,3 \\
\hline Otros usos & 9,9 & 8,3 & 7,1 & 23,8 & 0,0 & 41,7 \\
\hline Electricidad & 7,7 & 2,3 & 4,8 & 0,0 & 25,0 & 0,0 \\
\hline Laboral & 0,0 & 2,3 & 2,4 & 0,0 & 0,0 & 0,0 \\
\hline Otros & 0,0 & 3,8 & 2,4 & 0,0 & 6,3 & 0,0 \\
\hline Comunicaciones por congreso & $\mathbf{1 8}$ & $\mathbf{2 6}$ & $\mathbf{2 8}$ & $\mathbf{2 1}$ & $\mathbf{1 6}$ & $\mathbf{1 2}$ \\
\hline
\end{tabular}

Fuente: Société technique de l'industrie du gaz en France, Table des matières contenues dans les Comptes Rendus, 1874-1906, Paris, Imprimerie de la Société de publications periodiques, 1907; Compte rendu du congrès, 1914, 1929, 1938. Elaboración propia.

El segundo gran foco de atención lo presentan los sistemas de distribución, con especial hincapié en el control de las fugas ( $y$ del fraude) en las conducciones y las ventajas e inconvenientes de los distintos tipos de contadores (hidráulicos, secos, etc.). Los diferentes usos del gas constituyeron el tercer, y creciente, gran grupo de reflexión. Dentro de ellos destaca el alumbrado, por su carácter predominante en esa época. No obstante, resulta significativa la progresiva presencia en las discusiones técnicas de otros tipos de usos, como la calefacción, cocinas, motores, etc., estimula-

\section{LA PRESENCIA DE LA EUROPA LATINA EN LA SOCIÉTÉ TECHNIQUE}

La presencia de profesionales de la Europa latina en la asociación se mantuvo relativamente estable durante el período analizado. Constituían, en buena medida, el núcleo duro de la misma, con la lógica hegemonía de Francia, y un papel relevante de España (consecuencia de la fuerte influencia francesa en su industria del gas), algo menos de Italia (a causa de la relevante presencia de capital alemán y suizo en su sector gasista) y un ca- 
rácter casi testimonial de Portugal (debido a la escasa implantación del gas y al fuerte peso del capital británico). Dada la imposibilidad de ahondar en el perfil de todos ellos, nos limitaremos a bosquejar los rasgos y particularidades generales de los de España, Italia y Portugal, en especial de la primera, por su mayor peso en la Société Technique.

En las empresas españolas el número de representantes del país en la asociación era dominante (18 de 27 miembros computados en 1895). En cambio, en Italia y en Portugal ese año todos los socios eran extranjeros, aunque durante el primer tercio del siglo XX los italianos hicieron acto de presencia de manera creciente, sobre todo en 1938. El protagonismo español se redujo coyunturalmente en 1914 (9 de 19 socios), pero volvió a recuperarse en 1929 (15 de 23) y en 1938 (20 de 27).

En general, la permanencia en la asociación era dilatada. En 1895 el promedio de los socios españoles era de 12,5 años, casi el mismo (13 años) que el de los extranjeros enrolados en compañías que operaban en suelo español, y muy similar al de los asociados que operaban en Italia (11 años). Con los años ese promedio se elevó sustancialmente, debido a la larga permanencia de algunos afiliados, si bien a finales de los años veinte se observa una renovación de los afiliados.

En la documentación no siempre se especifica la profesión del socio, y sí su cargo o función en la compañía de la que formaba parte. Pero la gran mayoría eran ingenieros, por lo general (aunque no se indique) industriales. En España, además del principal fabricante de contadores de gas del país, en 1895 figuran 6 directores y un director general, de las fábricas de gas de Barcelona (La Propagadora del Gas), Gijón, Málaga, Murcia, Santander y Valencia. El resto ejercían como ingenieros o administradores, de empresas extranjeras (Compagnie Centrale d'éclairage par le gaz de Eugène Lebon, en Barcelona, Cádiz y Puerto de Santa María) o españolas (Sociedad Catalana para el Alumbrado por Gas), u otras funciones, como jefes de producción o inspectores técnicos. En cualquier caso, hay una clara preponderancia de ingenieros catalanes, síntoma del diferente grado de implantación de la industria en Cataluña y el resto de España. En Italia no es posible distinguir con tanta claridad un modelo regional dominante, aunque sí hay un claro sesgo en su localización hacia la fachada septentrional del país, en fábricas dirigidas al principio por ingenieros extranjeros que, con el paso del tiempo, cedieron su testigo a técnicos italianos, formados generalmente en universidades también del norte.
Entre los técnicos catalanes podemos destacar a José Mansana Terrés, miembro de la Société desde 1883 , y una de las figuras clave en la industria española del gas. Graduado por la Escuela de Ingenieros Industriales de Barcelona en 1889, además de abogado por la Universidad de Barcelona, fue propietario de 4 fábricas de gas en Cataluña. En 1892 sucedió a su padre (José Mansana Dordán), como administrador general de la gasista más importante del país, la Sociedad Catalana para el Alumbrado por Gas, desempeñando el puesto hasta su fallecimiento en 1934, además de desplegar una intensa actividad empresarial (Fàbregas, 2018, pp. 123-125).

Antonio Rovira i Borrell (miembro desde 1882) fue socio fundador de La Propagadora del Gas de Gràcia (1853), y director-gerente desde 1859 hasta su fallecimiento (1904). La empresa operó en el entorno de Barcelona, hasta su venta en 1883 a Eugène Lebon, abandonando el área en 1884 para extenderse por el Maresme, implantando o ampliando factorías: construyó la fábrica de Premià de Mar (1883) y compró las de Badalona (1884) y Terrassa (1886). Todo este patrimonio fue absorbido por La Catalana en 1913 (Arroyo, 1992, p. 63). También se encontraba afiliado a la asociación (desde 1883) Vicente Borrell, accionista y director industrial de la sociedad. Raimond Vilaclara $\mathrm{i}$ Dardañá, otro director-gerente (entre 1889 y 1912) de La Propagadora, y yerno del principal accionista (Antonio Rovira) se afiliaría en 1900.

Juan Gatell de Lomaña (asociado desde 1886), ingeniero industrial por la Escuela de Barcelona (1884), perteneciente a Lebon et Cie, fue director de la fábrica de gas de Almería (1886-1898), siendo nombrado en 1898 director de las de Cádiz y Puerto de Santa María (Fernández-Paradas, 2015, p. 111). Fue presidente del consejo y director-gerente de Gas y Electricidad de San Fernando, hasta su fallecimiento en 1931 (Sánchez Ruiz y Sánchez Miñana, 2017).

Federico Ciervo Pérez (asociado desde 1883), formado en Inglaterra, fue un fabricante de contadores y aparatos para gas y agua y otros productos metalúrgicos, a través de la casa Ciervo, creada en 1855 en Barcelona por el ingeniero mecánico inglés William Richards. Tras la salida de este en 1861 se hicieron cargo de ella, con capital de una destacada familia catalana, los Gil, constituyendo F. Ciervo y Compañía (desde 1873, Federico Ciervo y Cía), que se convirtió en un referente de la industria auxiliar del gas. Desde 1885 contó con la colaboración de dos de sus hijos, ingenieros industriales por la Escuela de Barcelona, dedicándose también a la construcción de fábricas de gas y diversificando la produc- 
ción hacia instalaciones eléctricas. Fue uno de los fundadores de la Compañía Española del Alumbrado por Gas de San Fernando, S.A. (1878), que en 1900 amplió su actividad al ramo eléctrico y en 1920 se convirtió en Gas y Electricidad de San Fernando, S.A. También fue socio de Alumbrado por Gas, José Piñol y Cía. de Granollers (Sánchez Miñana y Sánchez Ruiz, 2020, pp. 166-169).

César Santomá Allaigne, ingeniero industrial químico, doctor en Ciencias Físicas por la Universidad de Barcelona, y profesor de la Facultad de Ciencias de la Universidad de Valencia, fue director de la fábrica de gas valenciana, entre 1871 y 1924 . Era uno de los miembros más antiguos de la Société (desde 1874) y compartía destino con otros directores de fábricas del grupo Lebon en España: además del citado Juan Gatell, Rafael Caruana y Sánchez Plazuelos del Pulgar, director de las fábricas de Cádiz y Puerto de Santa María en 1886-1897 (miembro desde 1886); Alejandro de Martínez, de la de Murcia desde 1868 y Cartagena en 1869 (afiliado desde 1874), al que sucedería su hijo, con igual nombre, hasta 1924; Luís Quintanilla (miembro desde 1882) y Pedro de Zubeldia y Herrero (miembro desde 1926), de la de Santander en 1881-1898 y 1923-1937, respectivamente (Lebon et Cie, 1947, pp. $103,116,125,128,133-134)$. A estos se añadirían los ingenieros de la multinacional que trabajaban en España. Stanislas Smolinski, ingeniero civil, director de la gasista de Santander, quien fue director también de las fábricas de gas del grupo en Cartagena (1871) y Granada (1882), y mediada la década dirigió la factoría de Sanlúcar de Barrameda, propiedad de la Franco-Belge, Roberto Lesage et Cie (García de la Fuente, 1998, p. 201). La nómina se completa con Paul Delinon (Barcelona, socio desde 1874), Alfred Toussaint (Barcelona, 1886) o Léon Figuier (Cádiz, 1889).

En Galicia destaca una excepción en el perfil general, José de la Gándara Cividanes, graduado en la Academia Militar de Ingenieros de Guadalajara (1903). Compaginó su profesión militar con el asesoramiento a ayuntamientos y empresas en temas de infraestructuras urbanas. Ingeniero director de la Electra Popular de Vigo y Redondela desde 1921, fue nombrado ingeniero director de la Sociedad General Gallega de Electricidad en 1926, y miembro del Consejo de administración de las Fábricas Coruñesas de Gas y Electricidad en 1931, dirigiendo los procesos de fusión del conglomerado gasista-eléctrico gallego anterior a la Guerra Civil (Carmona, 2016, p. 93).

Los extranjeros, franceses en su gran mayoría, actuaban como directores, gerentes e ingenieros jefes, en las compañías de su país que operaban en Barcelona, Madrid (Compagnie Madrilène du gaz), A Coruña o
Zaragoza (Societé pour l'eclairage des villes de Biarritz et Saragosse). También en Italia y Portugal dominaban los directores e ingenieros franceses, en las fábricas de Bolonia, Livorno, Milán, Roma, Lisboa y Oporto, pero también había algún ingeniero perteneciente a sociedades gasistas belgas y alemanas.

Destaca la figura del ingeniero francés François Saunier Goubard (socio desde 1877), director de la fábrica de gas de Gijón (1870) de la concesionaria Menéndez, Valdés y Compañía. En 1871 instaló la fábrica de esa compañía en Santiago de Compostela (García, 2010, p. 21). En 1884 construyó la fábrica de Vigo y en 1888-1908 fue director de la de A Coruña, pertenecientes ambas a la Société Anonyme d'Eclairage, de Chauffage et de Force Motrice des villes de La Corogne et Vigo. Desempeñó un papel clave como uno de los impulsores iniciales del sector eléctrico gallego, ya que participó como accionista o asesor en varias empresas del ramo (Carmona, 2016, p. 38). Desde Gijón, también era afiliado (desde 1882) Eduardo Menéndez Valdés, director gerente de Menéndez Valdés y Compañía.

En vísperas de la guerra europea, la nómina de adheridos localizados en territorio español no sufrió sustanciales alteraciones, si exceptuamos que algunas de las firmas habían comenzado a diversificar el negocio, compatibilizando el gas y la electricidad. Se incorporaron algunos afiliados nuevos, en sustitución de directores anteriores, principalmente en las citadas fábricas barcelonesas. Lo más Ilamativo es la entrada de varios directores franceses, en las instalaciones de Ciudad Real, A Coruña, Vigo, Madrid, Málaga y Zaragoza, lo que formaba parte del proceso de adiestramiento que a menudo realizaban los ingenieros enrolados en las multinacionales de ese país. En cambio, se vislumbra un gradual protagonismo de los ingenieros italianos como responsables de las explotaciones de gas en varias localidades (Biella, Milán, Módena, Roma y Venecia), aunque prevalecía el rol de franceses o belgas (Florencia o Verona). En la antesala de la Segunda Guerra Mundial todo vestigio foráneo en la industria trasalpina del gas había desaparecido, sobresaliendo algunas figuras locales, que aparecen como socios de honor. Destacan Michelangelo Böhm, ingeniero por el Politécnico de Milán y uno de los principales expertos en gas del país, que llegó a ser presidente de la Union internationale de l'industrie du gaz. Ernesto Sospisio, emprendedor en el campo de los hidrocarburos, presidente, entre otras entidades, de la Banca Triestina, y presidente de la Associazione Italiana delle Industrie Gas e Aqua. Y Ugo Dellacasa, ingeniero de la Societá Milanese per l'Industria del Gas, quien también presidió aquel organismo. 
La corriente nacionalizadora que impregnó la vida económica española durante los años veinte tuvo su manifestación en la industria gasista en forma de un retroceso de las posiciones del capital extranjero. Esto explica el crecimiento del número de ingenieros españoles participantes en la Société en 1929, la mayoría de ellos encargados de gestionar sociedades con una vocación cada vez más marcadamente eléctrica y, por tanto, con un perfil competencial más en consonancia con el auge de la nueva energía. De ahí que su ingreso se produjese mediada la década, dentro de un escenario análogo al vivido en Italia, y muy similar asimismo a lo ocurrido con los nuevos técnicos franceses, desplazados en esos años a España (la compañía Lebon, y sus plantas en Barcelona, Cádiz, Málaga o Valencia).

\section{CONCLUSIONES}

La inversión exterior directa ha sido considerada tradicionalmente uno de los principales vehículos de transmisión internacional de la tecnología. Esta hipótesis se confirma plenamente en el caso de la industria gasista. Francia, país líder junto con Gran Bretaña en este sector, constituyó un potente foco emisor a través de sus empresas situadas principalmente en la Europa latina (España e Italia, en menor medida Portugal, de tradicional influencia británica).

No obstante, un elemento escasamente considerado ha sido el papel desempeñado por las asociaciones

\section{NOTAS AL FINAL}

1 Sobre la historia de esta asociación, Association technique de l'industrie du gaz en France: Les cent ans de l'Association technique de l'industrie du gaz en France (18741974), Laval (Mayenne), Imprimerie Barnéoud, 1974.

2 Para un perfil de los negocios de algunos de ellos, ver Fàbregas (2003, pp. 81-114).

3 Creado en 1928, publicará un boletín bibliográfico mensual de gran éxito. Por otro lado, pasará de enviar 250 documentos a los socios en ese año a 1.400 en 1938 . También se intensificará la traducción de trabajos extranjeros relevantes, generalmente publicados en forma de resumen en el Journal des usines a gaz. A partir de 1929 puso a disposición de los asociados las traducciones efectuadas por los ingenieros en su trabajo y dos años más profesionales en la diseminación del conocimiento técnico. El análisis de la Société Technique ha puesto de relieve la importancia de esas entidades. Resulta sintomática la coincidencia de la procedencia mayoritaria de sus socios, la Europa latina, con la presencia de compañías francesas de gas. Las vías utilizadas por esta entidad para la transmisión del conocimiento entre sus asociados fueron los congresos, tanto nacionales (internacionales de facto dada la importante presencia habitual de participantes no franceses) como internacionales, su influyente órgano oficial de difusión, el Journal des usines a gaz, su rica biblioteca, nutrida con las principales revistas y obras del sector, y sus reputados instrumentos de formación: semana gasista y Centre d'études supérieures gazieres.

Su evolución a lo largo del medio siglo anterior al inicio de la Segunda Guerra Mundial arroja luz sobre las cambiantes preocupaciones de la industria del gas, los debates científicos en su seno, así como, indirectamente, acerca del peso relativo de los distintos países en la tecnología del gas.

\section{AGRADECIMIENTOS}

Este artículo forma parte de los resultados del Proyecto I+D+I de Excelencia, PID2020-112844GB-100, "El gas en la Europa Latina: una perspectiva comparativa y global (1818-1945)", financiado por el Ministerio de Ciencia e Innovación del Gobierno de España y Fondos Feder.

tarde impulsó un cuerpo de traductores voluntarios. En 1936 editó una Guía práctica sobre la industria del gas, con 2.000 ejemplares agotados en poco tiempo.

4 Para ello incrementará el número de miembros del Comité a 27.

5 La ATG se encargará, dentro de la Union, del área de estandarización internacional de aparatos y métodos de ensayo (ATIGF, 1974, pp. 81-84).

6 El programa incluía, además de las técnicas de producción y distribución del gas, aspectos sociales, administrativos y de gestión empresarial. La metodología, eminentemente práctica, se desarrollaba a lo largo de tres sesiones de dos semanas cada una, completada con un viaje de estudios. El número de alumnos inicial fue de veinte. Entre 19301973 el número de alumnos fue de 1.004, 67 de ellos extranjeros 


\section{BIBLIOGRAFÍA}

Abramovitz, Moses (1979), "Rapid Growth Potential and its Realization: The Experience of the Capitalist Economies in the Postwar Period". En: Malinvaud, Edmond (ed.), Economic Growth and Resources, Proceedings of the Fifth World Congress of the International Economic Association, vol. 1, pp. 1-30.

Alayo Manubens, Joan Carles; Barca Salom, Francesc Xavier (2017), "Las técnicas de fabricación utilizadas en las fábricas de gas españolas (1842-1972)" y “Apéndice”. En: Bartolomé Rodríguez, Isabel, Fernández-Paradas, Mercedes y Mirás Araujo, Jesús (eds.), Globalización, nacionalización y liberalización de la industria del gas en la Europa latina (siglos XIX-XXI), Madrid, Marcial Pons, pp. 141-171 y pp. 317-323.

Arroyo, Mercedes (1992), “"La Propagadora del Gas», de Gracia: articulación del territorio y administración municipal”, Ciudad y Territorio, 94, p. 61-77.

Berg, Maxine; Bruland, Kristine (1998), "Culture, Institutions and Technological Transitions". En: Berg, Maxine y Bruland, Kristine (eds.), Technological Revolutions in Europe: Historical Perspectives, Cheltenham, Edward Elgar, pp. 3-16.

Braunholtz, Walter (1963), The Institution of Gas Engineers: The First Hundred Years, 1863-1963, Hertford, Institution of Gas Engineers.

Buchanan, Robert A. (1986), "The Diaspora of British Engineering", Technology and Culture, 27 (3), pp. 501-524.

Cantwell, John (1990), "Historical trends in international patterns of technological innovation". En: Foreman-Peck, J. (ed.), New Perspectives on the late Victorian Economy: Essays in Quantitative Economic History, 1860-1914, Cambridge, Cambridge University Press, pp. 37-72.

Cardoso de Matos, Ana (2017), “A indústria de gás em Portugal: Uma primeira tentativa de comparação com Espanha (18481950)". En: Bartolomé Rodríguez, Isabel, Fernández-Paradas, Mercedes y Mirás Araujo, Jesús (eds.), Globalización, nacionalización y liberalización de la industria del gas en la Europa latina (siglos XIX-XXI), Madrid, Marcial Pons, pp. 75-95.

Carmona Badía, Xoán (2016), La Sociedad General Gallega de Electricidad y la formación del sistema eléctrico gallego (1900-1955), Barcelona, Fundación Gas Natural Fenosa.

Cubel, Antonio; Esteve, Vicente; Sanchis, Juan A.; Sanchis, María T. (2012), "Medio siglo de innovación y transferencia de tecnología en España, 1950-2000", Revista de Historia Industrial, 50, pp. 113-154.

De Mello Jr., Luiz R. (1997), “Foreign Direct Investment in Developing Countries and Growth: A Selective Survey", The Journal of Development Studies, 34 (1), pp. 1-34.

Edgerton, David (1999), "From Innovation to Use: ten (eclectic) theses on the history of technology", History and Technology, 16, pp. 1-26.

Fàbregas, Pere-A. (2003), La Globalización en el siglo XIX: Málaga y el gas, Sevilla, Ateneo de Sevilla - Universidad de Sevilla.

Fàbregas, Pere-A. (2018), Naturgy. 175 años de compromiso con la energía y la sociedad, Barcelona, Planeta.

Fernández-Paradas, Mercedes (2015), La industria del gas en Cádiz, Sabadell, Fundación Gas Natural Fenosa.
Franco, Rossella (1988), "Industrializzazione e servizi. Le origini dell'industria del gas in Italia", Italia contemporanea, 171, pp. 15-38.

García, Eduardo (2010), Luces de Gijón. El alumbrado público municipal (1834-2010), Gijón, Ayuntamiento de Gijón.

García de la Fuente, Dionisio (1998), La historia del gas en Granada. Del Gas Lebon al gas natural, Sevilla, Gas Andalucía.

Giuntini, Andrea (1997), "Il gas in Italia fra industria e servizio urbano dall'avvento dell'elettricità alla scoperta del metano". En: Biaggati, G., Giuntini, A., Mantegazza, A. y Rotondi, C., L'acqua e il gas in Italia. La storia dei servizi a rete, delle aziende pubbliche e della Federgasacqua, Milano, Franco Angeli, pp. 163-255.

Grelon, André (1993), “The training and career structures of engineers in France, 1880-1939". En: Fox, Robert y Guagnini, Anna (eds.), Education, Technology and Industrial Performance in Europe, 1850-1939, Cambridge, Cambridge University Press, pp. 42-64.

Hausman, Wiliam J.; Hertner, Peter; Wilkins, Mira (2008), Globa Electrification: Multinational Enterprise and International Finance in the History of Light and Power, 1878-2007, Nueva York, Cambridge University Press.

Helpman, Elhanan (2004), The Mystery of Economic Growth, Cambridge, Harvard University Press.

Inkster, Ian (1991), Science and Technology in History. An Approach to Industrial Development, London, Macmillan.

Inkster, lan (1998), "Lessons of the Past? Technology Transfer and Russian Industrialisation in Comparative Perspective", Science, Technology \& Society, 3 (2), pp. 307-333.

Lebon \& Cie. Un Centenaire 1847-1947, Paris, 1947.

Martykánová, Darina (2010), Los ingenieros en España y en el Imperio Otomano. Una historia comparada, tesis doctoral, Universidad Autónoma de Madrid.

Mokyr, Joel (1992), "Technological Inertia in Economic History", The Journal of Economic History, 52 (2), pp. 325-338.

Ortiz-Villajos, José M. (2006), “Patentes y desarrollo económico en la España contemporánea". En: Merger, Michèle (dir.), Transferts de technologies en Mediterranée, Paris, PUPS, pp. 53-68.

Pretel, David (2009), "Invención, nacionalismo tecnológico y progreso: el discurso de la propiedad industrial en la España del siglo XIX", Empiria. Revista de metodología de ciencias sociales, 18 , pp. 59-83.

Sáiz González, J. Patricio (2012), "Social Networks of Innovation in the European Periphery. Exploring Independent versus Corporate Patents in Spain circa 1820-1939", Historical Social Research / Historische Sozialforschung, 37 (4), pp. 348-369.

Sánchez Miñana, Jesús; Sánchez Ruiz, Carlos (2020), "Ciervo en la Barceloneta (1861-circa 1960): ascenso y declive de una empresa auxiliar de la industria del gas, primera fábrica de contadores de España". En: Bartolomé Rodríguez, Isabel, Fernández-Paradas, Mercedes y Mirás Araujo, Jesús (eds.) Cercanas pero distintas: la desigual trayectoria de la industria del gas en las regiones del sur de Europa (siglos XIX-XX), Madrid, Marcial Pons, pp. 165-182. 
Sánchez Ruiz, Carlos; Sánchez Miñana, Jesús (2017), “Cincuenta años de suministro eléctrico a San Fernando (Cádiz). De la fábrica de gas a la Compañía Sevillana (1899-1948)". En: Capel Sáez, Horacio, Zaar, Miriam H. y Vasconcelos Pereira Junior, Magno (eds.), La electrificación y el territorio: historia y futuro, Barcelona, Universitat de Barcelona.

Thomas, Russell (2018), "The development of the manufactured gas industry in Europe". En: Craig, Jonathan, Gerali, Francesco, Macaulay, Fiona y Sorkhabi, Rasoul (eds.), History of the European Oil and Gas Industry, London, The Geological Society, pp. 137-164.

Tomory, Leslie (2011), "Gaslight, distillation, and the Industrial Revolution", History of Science, 49 (4), pp. 395-424.

Tomory, Leslie (2012), Progressive Enlightenment: The Origins of the Gaslight Industry, 1780-1820, Cambridge, MA: MIT Press.

Van Pottelsberghe, Bruno; Lichtenberg, Frank (2001), "Does Foreign Direct Investment Transfer Technology Across Borders?", The Review of Economics and Statistics, 83 (3), pp. 490-497.

Williot, Jean-Pierre (2005), "De la naissance des compagnies à la constitution des groups gaziers en France (années 18201930)". En: Paquier, Serge et Williot, Jean-Pierre (dirs.), L'industrie du gaz en Europe aux XIXe et XXe siècles. L'innovation entre marchés privés et collectivités publiques, Bruxelles, P.I.E.-Peter Lang, pp. 53-64.

Williot, Jean-Pierre (2006), "La diffusion de la technologie gazière française dans le bassin méditerranéen: de la construction des usines à gaz à la mise en place des réseaux de gaz naturel (années 1840-1980)". En: Merger, Michèle (ed.), Transferts de technologies en Méditerranée, Paris, Presses Universite Paris-Sorbonne, pp. 207-219.

Williot, Jean-Pierre; Paquier, Serge (2005a), “Origine et diffusion d'une technologie nouvelle au XIXe siècle". En: Paquier, Serge et Williot, Jean-Pierre (dirs.), L'industrie du gaz en Europe aux XIXe et $X X^{e}$ siècles. L'innovation entre marchés privés et collectivités publiques, Bruxelles, P.I.E.-Peter Lang, pp. 21-51.

Williot, Jean-Pierre; Paquier, Serge (2005b), "Stratégies entrepreneuriales et évolution des marchés des années 1840 aux années 1930". En: Paquier, Serge et Williot, Jean-Pierre (dirs.), L'industrie du gaz en Europe aux XIXe et XXe siècles. L'innovation entre marchés privés et collectivités publiques, Bruxelles, P.I.E.-Peter Lang, pp. 53-64.

Woo, Jaejoon (2009), "Productivity growth and technological diffusion through foreign direct investment", Economic Inquiry, 47(2), pp. 226-248. 